\title{
Algunos aspectos clínicos y epidemiológicos de la isosporosis intestinal. Estudio en 26 pacientes pediátricos de la ciudad de Antofagasta
}

\author{
Dres. Hernán Sagua*, Manuel Zamorano**, Julia Soto*, Magaly Raby* y Alejandro Fuentes*
}

Las enfermedades del aparato digestivo constituyen un importante problema de salud pública en Chile, principalmente entre la población infantil.

En este grupo humano las infecciones por protozoos enteroparásitos han sido ampliamente estudiadas, dada la alta frecuencia con que se presentan (Rubio y col., 1962; Bull y col., 1964; Puga y col., 1973; Noemí y col., 1977), señalán dose en todas estas investigaciones a Giardia lamblia como el agente etiológico más frecuente. Sin embargo, la magnitud del problema hace necesaria la constante revisión y estudio de los factores y agentes que inciden en la producción de nuevos casos.

$\mathrm{La}$ isosporosis humana, que es una infección enteroparasitaria producida por Isospora belli Wenyon, 1925 o Isospora hominis (Railliet y Lucet, 1891), Wenyon 1923, parásitos de patogenicidad condicionada y de localización primaria en el intestino del hombre, también es una afección del aparato digestivo, que periódicamente se presenta como brotes epidémicos y estacionales, característicos de esta parasitos is (Jarpa y col. 1966; Dodds y col., 1955).

Actualmente las dos especies causantes del cuadro están siendo intensamente estudiadas, lo que ha permitido aclarar el ciclo biológico de 1. hominis (Rommel y col., 1972). Para I. belli, la especie más frecuente y patógena, sólo se co-

\footnotetext{
* Grupo de Microbiología y Parasitología.

* Grupo de Especialidades, Departamento de Ciencias de la Salud, Universidad de Chile - Sede Antofagasta.
}

noce el ciclo intestinal en el hombre y el fecalismo como fuente de infección (Niedmann, 1963; Jarpa y col. loc. cit.).

Entre los infectados por 1 . belli se observan dos grupos bien definidos: uno asintomático, tal vez el más numeroso en la edad pediátrica, y otro grupo sintomático que se caracteriza por presentar el síndrome diarreico recidivante, pudiendo éste constituir por sí sólo todo el cuadro clínico, y está integrado principalmente por niños bien nutridos y por adultos (Faust y col., 1961, Dodds y col., loc. cit.; Jarpa y col. loc. cit.).

Debido a estos antecedentes clínicos y epidemiológicos, suelen presentarse algunas dificultades para su manejo en la clínica diaria.

En los últimos tres años la Unidad de Parasitología del Departamento de Ciencias de la Salud de la Universidad de Chile, Sede Antofagasta, ha realizado encuestas coproparasitológicas entre los escolares y preescolares de la ciudad, detectando 8 casos de isosporosis en niños asintomáticos. Igualmente, durante un brote epidémico de gastroenteritis que se presentó en 1977 (Sagua y col.), se encontraron 18 nuevos casos en niños, los que fueron evaluados y examinados clínicamente, investigándose la serología para infecciones entéricas y toxoplasmósicas.

La presente comunicación tiene como propósito dar a conocer un análisis clínico y epidemiológico de estos 26 casos pediátricos de isosporosis intestinal por $I$. belli encontrados en Antofagasta. 


\section{MATERIAL Y METODO}

Casuística constituida por 26 pacientes ambulatorios de ambos sexos, de 2 a 15 años, atendidos por la Unidad de Parasitología, del Departamento de Ciencias de la Salud, de la Universidad de Chile, Sede Antofagasta entre 1976 y 1978, diagnosticándose por exámen parasitológico de deposiciones, según el método de Telemann modificado (Silva y col., 1963), ooquistes de Isospora bellu. 18 de estos casos se encontraron durante un brote epidémico de gastroenteritis. Los 8 pacientes restantes se pesquisaron en encuestas enteroparasitológicas anuales.

En una ficha especial se registraron las observaciones epidemiológicas y clínicas de cada caso.

El estudio epidemiológico constó de la información entregada por el paciente y sus familiares y de una visita domiciliaria. Además se investigó el agua y las hortalizas que se riegan con la producción de una planta de agua industrial de la ciudad.

El estudio clínico se basó en una anamnesis cuidadosa, examen físico, conducta terapéutica si la hubo, evolución y exámenes de laboratorio (Hemograma y recuento absoluto de eosinófilos, reacción de Widal, serología para toxoplasmosis ${ }^{1}$ ) para completar la investigación.

\section{RESULTADOS}

De los 26 casos estudiados, $16(61,53 \%)$ correspondían al sexo masculino y $10(38,47 \%)$ al sexo femenino. Las edades fluctuaron entre los 2 y 15 años, siendo su distribución homogénea entre preescolares $(46 \%)$ y escolares $(54 \%)$ Tabla 1 .

\section{Tabla 1}

ISOSPOROSIS INTESTINAL. DISTRIBUCION POR SEXO Y EDAD DE 26 PACIENTES PEDIATRICOS. ANTOFAGASTA, CHILE, 1979

\begin{tabular}{|c|c|c|c|c|c|}
\hline \multirow{3}{*}{ Edad } & \multirow{3}{*}{ (años) } & \multicolumn{4}{|c|}{$S E X O$} \\
\hline & & \multicolumn{2}{|c|}{ Masculino } & \multicolumn{2}{|c|}{ Femenino } \\
\hline & & $N$ & $\%$ & $N^{\circ}$ & $\%$ \\
\hline 2 & 5 & 6 & 23 & 6 & 23 \\
\hline 6 & $-\quad 14$ & 10 & 38 & 4 & 15 \\
\hline
\end{tabular}

\footnotetext{
${ }^{1}$ Departamento de Microbiología y Parasitología, Facultad de Medicina Santiago Norte. Universidad de Chile.
}

El estudio hematológico completo se practicó en 21 pacientes, destacando que el mayor número de casos se agrupa con valores de eosinofilia relativa entre $1.5 \%$ (10) y de eosinofilia absoluta entre $100-350 \times \mathrm{mm}^{3}(9)$. Tabla 2.

\section{Tabla 2}

ISOSPOROSIS INTESTINAL. EOSINOFILIA RELATIVA Y ABSOLUTA EN 21 PACIENTES PEDIATRICOS. ANTOFAGASTA, CHILE. 1979

\begin{tabular}{cccc}
\hline$N^{0}$ casos & $\begin{array}{c}\text { Eosinofilia } \\
\text { Relativa }(\%)\end{array}$ & $N^{\circ}$ casos & $\begin{array}{c}\text { Eosinofilia } \\
\text { Absoluta }\left(\mathrm{mm}^{3}\right)\end{array}$ \\
\hline 10 & $1-5$ & 9 & $100-350$ \\
6 & $6-25$ & 8 & $351-1000$ \\
5 & 2645 & 4 & $1001-2000$ \\
\hline
\end{tabular}

Eosinofilia absoluta aumentada sólo se observó en 4 casos entre 1.080 y 1.104 eosinófilos $\times \mathrm{mm}^{3}$ (Jarpa y col., loc. cit.; Sagua y col., loc. cit.).

El estudio serológico para gérmenes entéricos, por reacción de Widal, se practicó en 23 pacientes, resultando negativo en 22. En un sólo caso se obtuvo un título positivo inactivo por no presentar modificación al efectuar la reacción de control, que sólo fue posible aplicarla en 11 de los pacientes en estudio. Tabla 3.

Tabla 3

ISOSPOROSIS INTESTINAL. REACCION DE WIDAL EN 26 CASOS PEDIATRICOS. ANTOFAGASTA, CHILE. 1979 CHILE. 1979

\begin{tabular}{lcc}
\hline Reacción de Widal & $\begin{array}{c}\text { Test diagnóstico } \\
N^{\circ} \text { casos }\end{array}$ & $\begin{array}{c}\text { Test control } \\
N^{\circ} \text { casos }\end{array}$ \\
\hline Serologia negativa & 22 & 10 \\
Seropositivo activo & 0 & 0 \\
Seropositivo inactivo & 1 & 1 \\
\hline
\end{tabular}

Respecto a las reacciones de fijación de complemento (RFC) y hemaglutinación (RHA) para toxoplasmosis se practicaron en 18 pacientes, resultando en todos ellos negativa la RFC y sólo en 2 casos positiva la RHA con título sin significación clínica (1:16), catalogándoseles como seropositivos inactivos. Tabla 4.

El examen parasitológico de deposiciones permitió diagnosticar además de la isosporosis por Isospora belli, 11 casos de poliinfección por otros parásitos, destacando la mayor incidencia de Giardia lamblia $(15,3 \%)$ y de Entamoe- 
ba coli $(26,9 \%)$. Los cristales de Charcot-Leyden se detectaron en $23(88,4 \%)$ pacientes. Tabla 5.

En la evaluación clínica de los casos sintomáticos ${ }^{11}$, se encontró que el síndrome diarreico prolongado, la anorexia y el dolor abdominal eran la sintomatología predominante. Fiebre se presentó habitualmente al comienzo del cuadro con temperaturas que no superaron los $38,5^{\circ}-39^{\circ} \mathrm{C}$ en 6 pacientes. La condición de asintomáticos (diagnosticada la I. belli por encuesta escolar) se observó en 15 pacientes. El examen físico en todos los casos estudiados fue normal. Tabla 6.

Frente a las condiciones de saneamiento ambiental y la higiene individual, factores predisponentes para las enteroparasitosis, se puede apreciar que el saneamiento ambiental con la condición de bueno y malo se observó en 10 y 11 casos respectivamente, en cambio la higiene individual fue buena en 12 pacientes y mala en 8. Tabla 7.

El estudio parasitológico que se practicó a las aguas de la planta industrial y a las hortalizas que se riegan con ellas, nos permitió detectar la presencia de quistes de enteroparásitos y comensales humanos y cristales de CharcotLeyden. Tabla 8.

Tabla 4

ISOSPOROSIS INTESTINAL. SEROLOGIA PARA TOXOPLASMOSIS EN 26 PACIENTES

PEDIATRICOS. ANTOFAGASTA. CHILE 1979

\begin{tabular}{lcc}
\hline $\begin{array}{l}\text { Actwidad } \\
\text { Inmunológica }\end{array}$ & $\begin{array}{c}\text { RHA } \\
N^{\circ} \text { casos }\end{array}$ & $\begin{array}{c}\text { RFC } \\
\text { casos }\end{array}$ \\
\hline Serología negativa & 16 & 18 \\
Seropositiva activa & 0 & 0 \\
Seropositivo inactivo & 2 & 0 \\
\hline
\end{tabular}

Tabla 5

ISOSPOROSIS INTESTINAL. EXAMEN

PARASITOLOGICO DE DEPOSICIONES EN 26

PACIENTES PEDIATRICOS. ANTOFAGASTA, CHILE 1979

\begin{tabular}{lrr}
\hline PARASITOS & $N^{\circ}$ & $\%$ \\
\hline Isospora belli & 26 & 100,0 \\
Giardia lamblia & 4 & 15,3 \\
Entamoeba histolytica & 1 & 3,8 \\
$\quad$ COMENSALES & & \\
Entamoebacol & 7 & 26,9 \\
Endolimax nana & 6 & 23,0 \\
$\quad$ CRISTA LES & & \\
Charcot-Leyden & 23 & 88,4 \\
\hline
\end{tabular}

Tabla 6

ISOSPOROSIS INTESTINAL. FRECUENCIA DE LA SINTOMATOLOGIA PREDOMINANTE EN 26 PACIENTES PEDIATRICOS. ANTOFAGASTA, CHILE, 1979

\begin{tabular}{lcc}
\hline Signos $y$ sintomas & $N^{\circ}$ casos & $\%$ \\
\hline Sindrome diarreico & 11 & 42,30 \\
Anorexia & 8 & 30,76 \\
Dolor abdominal & 7 & 26,92 \\
Fiebre & 6 & 23,07 \\
Pérdida de peso & 6 & 23,07 \\
Meteorismo & 5 & 19,23 \\
Asintomático & 15 & 57,69 \\
\hline
\end{tabular}

Tabla 7

ISOSPOROSIS INTESTINAL. CLASIFICAACION DE 26 PACIENTES PEDIATRICOS, SEGUN SANEAMIENTO AMBIENTAL E HIGIENE INDIVIDUAL. ANTOFAGASTA, CHILE, 1979

\begin{tabular}{|c|c|c|c|c|}
\hline \multirow[t]{2}{*}{ Condición } & \multicolumn{2}{|c|}{$\begin{array}{c}\text { Saneamiento } \\
\text { ambiental }\end{array}$} & \multicolumn{2}{|c|}{$\begin{array}{l}\text { Higiene } \\
\text { indwidual }\end{array}$} \\
\hline & $N^{\circ}$ & $\%$ & $N^{\circ}$ & $\%$ \\
\hline Bueno & 10 & 38,46 & 12 & 46,15 \\
\hline Regular & 5 & 19.23 & 6 & 23,07 \\
\hline Malo & 11 & 43,30 & 8 & 30.76 \\
\hline
\end{tabular}

Tabla 8

ISOSPOROSIS INTESTINAL. EXAMEN PARASITOLOGICO EN 24 MLESTRAS DE AGLA DE LA PLANTA INDUSTRIAL. ANTOFAGASTA, CHILE, 1979

\begin{tabular}{|c|c|c|c|}
\hline $\begin{array}{l}\text { Agua tratada } \\
\text { cruda }\end{array}$ & $\begin{array}{c}N^{\circ} \text { de } \\
\text { muestras }\end{array}$ & $\begin{array}{l}\text { Agua tratada } \\
\text { clorada }\end{array}$ & $\begin{array}{c}N^{\infty} \text { de } \\
\text { muestras }\end{array}$ \\
\hline PARASITOS & & RASITOS & \\
\hline Giardia lamblia & 2 & Giardia lamblia & 1 \\
\hline COMENSALES & & OMENSALES & \\
\hline Endolimax nana & 7 & Endolimax nana & 7 \\
\hline Entomoeba colt & 6 & Entamoeba coli & 3 \\
\hline Chilomastix mesnili & 1 & Chilomastix mesnili & 1 \\
\hline CRISTALES & & RISTALES & \\
\hline Charcot-Leyden & 2 & Charcot-Leyden & 1 \\
\hline
\end{tabular}




\section{COMENTARIO}

La isosporosis es una entidad mórbida que actualmente es objeto de múltiples investigaciones a nivel mundial, tanto en el plano clínico como biológico, tendientes a aclarar muchos de los aspectos empíricos conque ha permanecido esta parasitosis del hombre. Sin embargo, pese a los esfuerzos, aún no existen sustancias quimioterápicas específicas para su tratamiento. al igual que aún persisten dudas con el ciclo evolutivo de $I$. belli. En este aspecto, no ocurre lo mismo con I. hominis, de menor prevalencia humana; pero cuyo ciclo biológico ha sido aclarado en los últimos años, correspondiendo la infección humana a una forma de resistencia de coccidios del género Sarco cystis, propios del vacuno y el cerdo (Rommel y col., loc. cit.).

Estos antecedentes nos han movido a anal1zar nuestra casuística de 26 pacientes pediátricos, estudiados 18 de ellos durante un brote epidémico estacional típico de la parasitosis y pesquisándose los 8 casos restantes mediante encuestas entre preescolares y escolares, que en los últimos 3 años ha realizado la Unidad de Parasitología en la ciudad de Antofagasta.

Es de interés señalar que Antofagasta $\left(23^{\circ} 40^{\prime}\right.$ lat. Sur), es una ciudad puerto, con clima costero-desértico, lo cual limita la proliferación de algunos enteroparásitos, aun cuando la escasez de agua potable y deficiencias de saneamiento básico en algunos sectores de la ciudad, contribuyen a mantener tasas altas de infección por protozoos entéricos, principalmente a nivel infantil (Soto y col. 1978).

Del análisis ede los resultados, se observa que la infección afectó por igual a ambos grupos de niños, con porcentajes de $46 \%$ y $54 \%$ para preescolares y escolares respectivamente. Sin embargo la condición de sintomáticos se hizo más evidente en el grupo de los escolares, en los cuales además, el poliparasitismo fue la excepción.

En relación a los exámenes hematológicos, se confirma su utilidad diagnóstica, dado que la isosporosis es una de las pocas enteroparasitosis que presenta eosinofilias absolutas y relativas elevadas, aun cuando al parecer, en los niños los valores son inferiores a los observados entre los adultos (Jarpa y col., loc. cit.).
Ante cuadros diarreicos prolongados, subagudos y resistentes a la terapéutica habitual, se suele plantear la necesidad de descartar infecciones por gérmenes entéricos por lo cual se practicó a 22 pacientes la reacción de Widal. No se encontró título significativo de actividad inmunológica específica. Salvo en un caso que se obtuvo título de serología positiva inactiva para salmonellosis Para A de 1:40. Tampoco se pudo comprobar respuesta antigénica cruzada. Al test control practicado a sólo 11 pacientes la situación se mantuvo sin variaciones.

La estrecha relación biológica de Toxoplasma gondii con los esporozoos del género lsos. pora, nos llevaron a buscar respuesta inmunológica específica o cruzada de los pacientes en estudio, sin embargo, las reacciones de fijación de complemento, que indica actividad toxoplasmósica actual y la reacción de hemaglutinación que persiste positiva por largos periodos cuando hay infección toxoplasmósica, se presentaron negativas en todos los pacientes en que se probaron, salvo en dos de ellos que presentaron título de 1:16 para RHA (serología positiva inactiva).

Lno de los aspectos que más nos ha llamado la atención en nuestro estudio es la condición de pacientes asintomáticos que presentó el $57,69 \%$ (15), de los niños, lo cual también lo habian observado Dodds y E.lsdon-Dew (1955), en igual tipo de pacientes.

Igualmente resulta interesante el hecho que en este grupo de asintomáticos. la poliinfección con protozoos parásitos y comensales alcanzó las cifras más altas, sin que lógicamente apareciera sintomatología atribuible a ellos (Tabla 5). La única explicación para este hecho, creemos que sería la mayor exposición a infecciones repetidas por protozoos, ya que estos pacientes provenian de un sector con deficiente saneamiento ambiental y baja condición socioeconómica y cultural.

En los pacientes sintomáticos, el cuadro clínico y la evolución de la enfermedad permitió establecer un criterio diagnóstico bastante preciso en el cual el síndrome diarreico, el cólico intestinal, la anorexia, la pérdida de peso y el fracaso de tratamientos previos con antidiarreicos habituales, se complementó siempre con el hallazgo de ooquistes de $I$. belli y cristales de 
Charcot-Leyden en el examen parasitológico de deposiciones.

En este grupo de pacientes sintomáticos, generalmente sólo se diagnosticó la $I$. belli, siendo todos los casos de sectores con buen saneamiento ambiental y de niveles socioeconómicos y culturales altos, además de presentar estado nutritivo normal. En este sentido estamos de acuerdo con Jarpa (1966) y Daiber (1963), en que clínicamente se puede plantear el diagnóstico de isosporosis intestinal.

Algunas observaciones en relación a la investigación epidemiológica aplicada a nuestra casuística, sólo nos permiten postular al fecalismo humano como la fuente de infección y concordar con otros autores en que la máxima frecuencia para los brotes epidémicos ocurre entre otoño-inviemo, apareciendo en el resto del año casos aislados que suelen pasar desapercibidos. En nuestra ciudad la utilización de aguas industriales para regar hortalizas contribuiría a mantener tasas de infección por protozoos parásitos y comensales que pudimos diagnosticar en ellas, aun cuando relacionado con Isosporas sólo encontramos cristales de Charcot-Leyden (Tabla 8).

En relación a las condiciones socioeconómicas y culturales, podemos concluir que se observa un claro predominio de pacientes sintomáticos entre los grupos de mayores ingresos y buen nivel cult ural.

\section{RESUMEN}

Se hace una revisión clínica y epidemiológica de 26 casos pediátricos de isosporosis intestinal por Isospora belli, diagnosticados por examen parasitológico de deposiciones, encontrados en estudios de prevalencia para enteroparásitos entre escolares y preescolares y, durante un brote epidémico de gastroenteritis (1977), que afectí a la población de Antofagasta.

En el grupo en estudio es significativa la condición de pacientes asintomáticos $(57,69 \%)$ para isosporosis intestinal.

Entre los sintomáticos, la diarrea (2 a 30 días), el cólico intestinal, la baja de peso y la anorexia fueron los sintomas predominantes.

En 10 de los infectados se diagnosticó además Giardia lamblia (4), Endolimax nana (6) y Entamoeba coli (7). En un solo paciente se pesquisó Entamoeba histolytica. Los cristales de Charcot-Leyden aparecieron al examen copromicroscópico en $23(\mathbf{8 8 , 4 \%})$ muestras.
Ed estudio clínico se complementó con exámenes de laboratorio que incluyeron hemograma, reacciones de aglutinación para gérmenes entéricos y reacciones para toxoplasmosis.

Se confirman algunas observaciones útiles en relación con la epidemiologia de la isosporosis en ninos.

\section{SUMMARY}

A clinical and epidemic revision of 26 pediatric cases of intestinal isosporosis by Isospore belli is done. They have been diagnosed by means of a parasitologic examination of defecations found in prevailing research for enteroparasits among school and preschool children and during the gastroenterit is epidemic outbreak (1977), which affected the population of Antofagasta.

The condition of asymptomatic patients for intestinal isosporosis in the group in observation is significant $(57.69 \%)$.

Among the symptomatic patients the diarrhea (2) to 30 days), the intestinal colic, loss of weight and anorexia were the predominant symptoms.

In 10 of the infected patients it was also diagnosed Giardia lamblia (4), Endolimax nana (6) and Entamoeba coli (7). Only one patient was detected to have Entamoeba histolytica. The crystals of Charcot-Leyden appeared in the copromicroscopic in 23 samples ( $88.4 \%$ ).

The clinical research was complemented with laboratory examinations such as blood counts, reactions of agglutination for enteric germs and reactions for toxoplasmosis.

Some useful observations in relation to the epidemiology of isosporosis in children are confirmed

\section{REFERENCIAS}

1 Bull, F., Oyarce, R. y Stehr, Y. 1964. Contribución al estudio de prevalencia de parasitosis intestinales en algunas poblaciones de la provincia de Concepción (con especial referencia a Entamoeba histolytica y otros protozoos intestinales). Bol. Chile. Parasit. 19: 110-114.

2 Daiber, A. Vuillemin, L. y Schluf, A. 1964. Isosporosis en el sur de Chile. Bol. Chile. Parasit. 19:11-15.

a Dodds, S.E. and Elsdon-Dew, R. 195j. Further observations on human coccidiosis in Natal. S. Agr. J. Lab. Clan. Med. 1: 104-109.

4 Faust, E.C., Giraldo, L.E., Coicedo, ('. and Bonjanten, R. 19:1. Human isosporosis in the Western Hemispher Am. Trop. Med. Hyg. 10:343-349.

3 Janpa, A. 1966. Coccidiosis humana. Biológica 39 : $3-26$.

- Niedmann, G. 1963. Elementos parasitarios en la mucosa del intestino delgado en biopsias de enfermos con isosporosis. Bol. Chile. Parasit. 18:22.

7 Noemí, I., Reyes, H., Astorga, B. Glaser, P., Mauro, G., Tobar, M. Muñoz, V. y Gottlieb, B. 1977. Primer Simposio Inter, Parasit. Santiago, Chile, pp. 86.

9 Puga, S. 1973. Protozoos y Helmintos intestinales en lactantes de la ciudad de Valdivia, Chile. Bol. Chile. Parasit. 28: 39-40. 
Rommel, M. and Heydom, A.O. 1972. Contribución al ciclo evolutivo de los Sarcosporidios. III Isospora hominis (Railliet y Lucet, 1891). Wenyon, 1923, una forma de resistencia de los sarcosporidios de vacunos y cerdos. Berl. Much. Tierazth. Wschr. 85:143-145.

11 Rubio, M. 1962. Encuesta enteroparasitológica en el Hospital de niños "Luis Calvo Mackenna" en Santiago. Consideraciones epidemiológicas sobre 270 casos. Bol. Chile. Parasit. 77:93-100.

12 Sagua, H., Soto, J., Délano, B., Fuentes, A. y Becker, P. 1978. Brote epidémico de isosporosis por
Isaspora belli en la ciudad de Antofagasta. Chile. Cionsideraciones sobre 90 casos diagnosticados en 3 meses. Bol. Chile. Parasit. 33:8-12.

1.s Silva, R., Artigas, J. y Galdames, M. 1963. El diagnóstico en amibiasis intestinal crónica por los métodos combinados de concentración por centrifugación con formol salino y éter, y el alcohol polivinilico. Bol. Chile. Parasit. 18:92-96.

14 Soto, J., Sagua, H., Délano, B. y Fuentes, A. 1978. Encuesta sobre enteroparasitosis en preescolares de la ciudad de Antofagasta. Bol. Hosp. "San Juan de Dios". $25: 33-35$. 